“C 2010 IEEE. Personal use of this material is permitted. Permission from IEEE must be obtained for all other uses, in any current or future media, including reprinting/republishing this material for advertising or promotional purposes, creating new collective works, for resale or redistribution to servers or lists, or reuse of any copyrighted component of this work in other works.” 


\title{
Impact of Soft Handover and Pilot Pollution on Video Telephony in a Commercial Network
}

\author{
Lu Chen, Faisal Mohd. Madani, Kumbesan Sandrasegaran, Riyaj Basukala \\ Centre of Real-time Information Networks, Faculty of Engineering and Information Technology \\ University of Technology \\ Sydney, Australia \\ Lu.chen-4@student.uts.edu.au
}

\begin{abstract}
Soft handover (SHO) provides better support for user mobility. The quality of service $(\mathrm{QoS})$ to the mobile wireless subscribers can also benefit from SHO. To analyze the impact of SHO on the circuit switched (CS) video telephony (VT); real-time measurements have been conducted on a commercial WCDMA mobile network in the CBD of Sydney, Australia. Live measurement provides a practical approach to evaluate performance of commercial network. The obtained SHO gain implies that better QoS can be achieved. Furthermore, the negative impact of pilot pollution on radio link quality has been demonstrated in this paper.
\end{abstract}

Keywords-Mobile networks; $3 G$ WCDMA; video telehpony (VT); soft handover (SHO); quality of service (QoS); pilot pollution

\section{INTRODUCTION}

One of the most attractive features of mobile communication is service continuity with user mobility. However, this feature is largely restricted by coverage and capacity issues in cellular network. Handover (HO) plays a fundamental role for supporting service continuity with user mobility within coverage limits while balancing traffic load between adjacent or overlapped cells [1].

In traditional handover (hard HO), e.g. handover in GSM, a session is interrupted [2], i.e. the original connection from the source base station (BS) is released before establishing a new connection at the target base station (BS). This is referred to as "break before make". The interruption introduced by hard HO, though small, could have an impact on service continuity.

In wideband code division multiple access (WCDMA), which is the most popular air interface for the 3rd generation mobile wireless communication (3G) network, a new handover mechanism has been introduced and named soft handover (SHO). One key difference of SHO, when compared to hard $\mathrm{HO}$, is the 'no interruption' characteristic of handover procedures, which allows the user equipment (UE) to maintain multiple active connections simultaneously with different BS's. This handover mechanism is also referred to as "make before break". Soft handovers are needed for CDMA based systems because CDMA based systems are interference limited systems. Without SHO's, a mobile moving from one cell to an adjacent cell will have to transmit at a high power to communicate with the source BS resulting in interference in the target cell.

In WCDMA, a UE is continuously measuring all received signals on Primary Common Pilot Channel (P-CPICH; simply referenced as $\mathrm{CPICH}$ ). An Active Set is a list of cells to which $\mathrm{UE}$ is simultaneously connected to [3]. If a $\mathrm{CPICH}$ signal has been measured and is above a threshold value (set by the network) for a period of time ('Time-To-Trigger'), this $\mathrm{CPICH}$ would be included into the Active Set, and the BS, which this $\mathrm{CPICH}$ belongs to can be used for the UE transmission. BS with $\mathrm{CPICH}$, which has been measured with reasonably strong signal but not satisfied the above criteria to be included in the Active Set, will be registered in Monitored Set, as candidates for being added into Active Set [3]. In this manner, UE is able to connect to cells, which are capable of providing higher radio link (RL) quality. Therefore, the advantages of SHO can be, in turn, helpful for improving the quality of service (QoS) for the mobile wireless subscribers [1].

Soft handover results in a diversity gain called soft handover gain or SHO gain. A large number of investigations have been carried out on analyzing and improving SHO gain based either on theory or on simulation results, e.g. in $[2,4]$. However, other publications have studied the SHO gain and SHO performance in operational WCDMA networks. An early effort has been carried out by OrangeUK [5]. They conducted measurement on the trial WCDMA network then verified that SHO gains in downlink were achieved by receiving transmissions from two BS (in two-way handover) with spatial diversity as well as by using RAKE antenna on the receiving side at UE. A more recent work, [6] gave evaluations of SHO algorithm implemented on a pre-commercial WCDMA network by measurements results. Yet, neither [5] nor [6] have carried out measurements on a commercial network i.e. in the same environment as experienced by live subscribers. The author of [8] has conducted measurements in live scenario and assessed the quality of multimedia services on WCDMA network. However, the studies in [8] were focusing on the optimization of network planning procedure.

On another hand, pilot pollution issue commonly exists in WCDMA network. The polluting situation arises due to the severe interference among multiple CPICH's in cell overlapping area. In Reference [9], $\mathrm{CPICH}$ interference is named as "Multiple Access Interference (MAI)", which has 
important impact on capacity loss. However, only the severe condition of MAI-pilot pollution has been studied in [6] as well as a focus in our paper. In the study of [6], pilot pollution had impact on the performance of SHO algorithm. However, the work in [6] has only considered the polluting effect caused by the strongest $\mathrm{CPICH}$ in Monitored Set.

In our work, the analysis of the impact of SHO gain and of pilot pollution on mobile network has been carried out by performing live measurements on a commercially deployed WCDMA network in the CBD of Sydney, Australia. A circuit switched (CS) video telephony (VT) session has been used during measurements. The focus of this paper is on analyzing the impacts of SHO and of pilot pollution on maintaining QoS during SHO procedure in real-time scenario.

In Section II, the setup information of our measurements is described. Measurement results and analyses are presented in Section III. Finally, Section IV summarizes our study.

\section{MEASUREMENTS}

\section{A. Measurement environment}

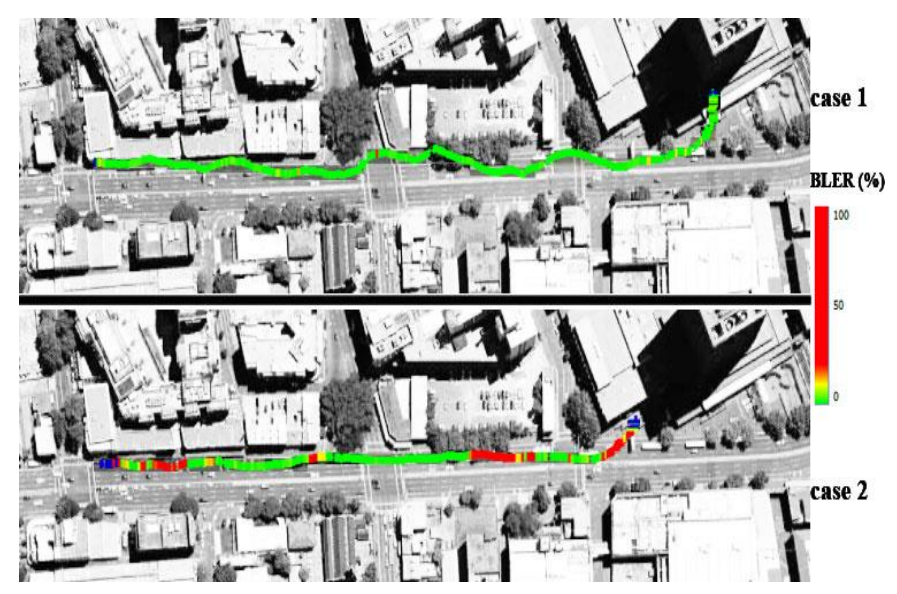

Figure 1. Downlink block error rate in test route. The upper picture is measured in live user case ( $\mathrm{SHO}$ enabled); the lower one is measured when

UE locked to Cell_1. The color set is shown on right-hand side.

The measurements are conducted on a commercial WCDMA mobile network implemented on the $2100 \mathrm{MHz}$ band in the CBD of Sydney, Australia. Specifically, the testing area was selected along the sideway of a main road of the city, while a little outside the city centre of high-rise core (surrounding by tall buildings) thus smaller density of user traffic and tall buildings. The start location of the measurement path was selected to be approximately at the edge of a $3 \mathrm{G}$ macro-cell and the end location was selected to be approximately at the middle of the cell. The specific cell will be referenced as 'Cell_1' or 'Scr.1' (corresponding scrambling code of 'Cell_1'), in the rest of this paper.

\section{B. Measurment tools}

The measurements were carried out by a commercial Nokia mobile handset of model 6720US (i.e. User Equipment (UE)), and UE Power Class 3. The real-time monitoring software,
Nemo Handy (Anite), has been embedded into the handset. Nemo Handy is used to display and record data of each measurement. The "lock to scrambling code" function in Nemo Handy is used when conducting measurements for the no-SHO scenarios. An independent GPS device has been connected to the handset via Bluetooth interface during measurements. The geographic information from the GPS can be integrated into the log files produced by Nemo Handy during measurements.

For post processing of the measurements, Nemo Analyze, MS Excel, MATLAB and Google Earth were used.

\section{Measured service}

The CS video telephony (VT) service was in use when performing live measurements. In WCDMA, the typical CS VT traffic is transferred on a symmetric-bidirectional dedicated channel (DCH) with 64kbps fixed bit rate [10]. At the UE used in this work, the CS VT has been compressed into 3GPP MPEG-4 format with a video resolution of $640 * 480$ pixels per frame, and streams at the frame rate of $15 \mathrm{fps}$.

VT was selected to be the service used in the measurements for three reasons. First, CS RT services are inherently sensitive to interruptions and errors, which may occur during handover processes. Second, VT is a multimedia service that emerged with 3G. Third, comparing to voice service (i.e. speech), VT has higher requirement of QoS (due to e.g. live video streams at higher bit rate and compressed picture frames are less robust to errors).

\section{Measurement cases}

Two measurement cases have been conducted in this paper. One is SHO case (Case 1) when UE is allowed to have SHO connection on the measured network. The other case (Case 2) is No-SHO case, when Nemo Handy has been configured to perform the functionality of 'Scrambling code locking' thus the UE can only be connected to a specific cell (Cell_1) (Table I.):

TABLE I. MEASUREMENT CASES

\begin{tabular}{|c|c|c|}
\hline Cases & Descriptions & Measurements \\
\hline Case 1 & $\begin{array}{c}\text { Live user scenario } \\
\text { (SHO) }\end{array}$ & $\begin{array}{c}\text { Case 1 1st run; } \\
\text { Case 1 2nd run }\end{array}$ \\
\hline Case 2 & $\begin{array}{c}\text { Cell locked scenario } \\
(\text { No-SHO) }\end{array}$ & $\begin{array}{l}\text { Case 2 1st run; } \\
\text { Case 2 2nd run }\end{array}$ \\
\hline
\end{tabular}

The pedestrian velocity measured by GPS is approximately $3.1 \mathrm{~km} / \mathrm{h}$, on average.

\section{E. KPI selection}

In SHO, the service continuity should be maintained, and the QoS is mainly affected by the radio frequency condition [8].

Thus, key performance indicators (KPI's) in the analysis are selected for indicating the radio frequency conditions and radio link quality of the UE:

- Call drop; 
- $\quad$ Downlink block error rate (BLER DL);

- Primary Common Pilot Channel (CPICH) Ec/No;

- Primary Common Pilot Channel (CPICH) RSCP;

- Downlink "power up" command and uplink transmission power of UE.

To illustrate the potential influence of pilot pollution, two additional KPIs are specified:

- The number of polluting pilots; and

- Total level of pilot pollution.

\section{ANALYSES RESULTS}

In this section, an analysis of the results is presented. A definition of the KPIs used in this work is presented.

\section{A. Soft handover performance}

\section{1) Call drop}

Call drop is a primary KPI of QoS to user. Possible causes of call drops can be from network's operational actions or due to poor RL quality. Technically, a number of factors may decrease RL quality hence cause call drops, such as handover failure, poor coverage, insufficient capacity, etc. [11]. In our measurements, call drop has not been obtained in either Case 1 or Case 2.

\section{2) Downlink block error rate (BLER DL)}

For an active call session, the analysis of downlink block error rate (BLER) is used for evaluating the overall quality of reception for UE, given that any physical layer errors occurred in transmission can be reflected by BLER.

The transmission errors will mainly affect the quality of video rather than voice when the received data is extracted and fed to the user applications [11]. An erroneous block in VT can be perceived by user as 'image distortion' or 'incontinuous image' [11]. References [2] and [10] have specified the BLER DL in VT should be lower than $1 \%$ at the receiver.

According to [12], BLER is computed after Radio Link (RL) combination in the Rake Receiver of the UE using the formulae given in (1).

$$
B L E R=\frac{\sum \text { number of received transport blocks in CRC error }}{\sum \text { number of total received transport blocks }}
$$

The CDF analysis of downlink transport channel BLER in both Case 1 and 2 is presented in Figure 2.

According to Figure 2, a much better BLER in Case 1 is obtained. In Case 1 , for $80 \%$ of the time no error has been measured on BLER, while only BLER values of $0-20 \%$ are obtained in measurements. The worst BLER value is approximately $20 \%$ based on average of two test runs in Case 1. In Case 2, the BLER value of $1 \%$ corresponds to a CDF of approximately 0.55 . Therefore the poor link quality situation (i.e. BLER $>1 \%$ ) have been obtained for approximately $45 \%$ of the session time and maximum BLER value is as high as $80 \%$.

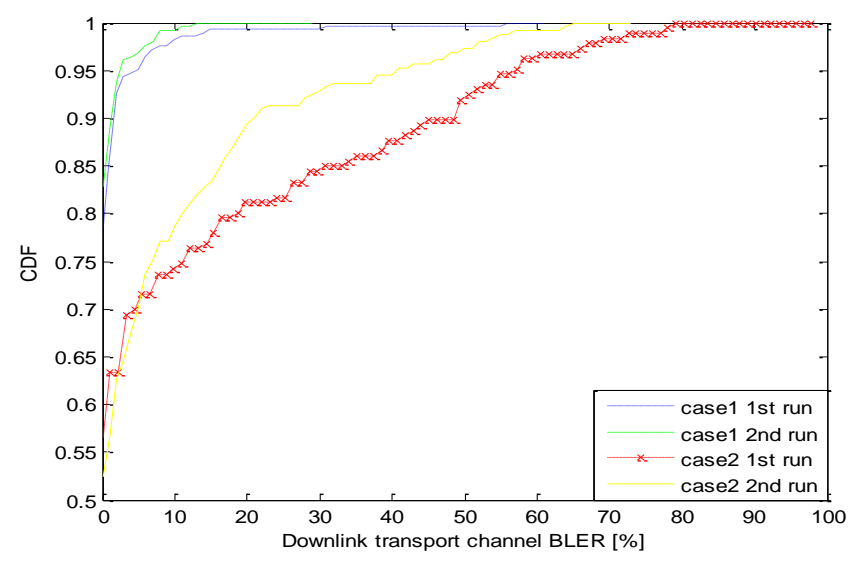

Figure 2. CDF of downlink transport channel BLER in each measurements when sho is enabled and disabled, respectively

\section{3) Primary Common Pilot Channel (CPICH) Ec/No}

The direct indicator of RL quality is the received $\mathrm{CPICH}$ $\mathrm{Ec} / \mathrm{No}$ by the UE. By definition in [12], $\mathrm{CPICH}$ Ec/No is the received energy per chip divided by power density in the band. Generally, stronger the received $\mathrm{CPICH}$ Ec/No at UE, lower the interference and noise level in the RL, hence better signal quality can be achieved, or less downlink transmission power from the BS is required for receiving similar quality signal.

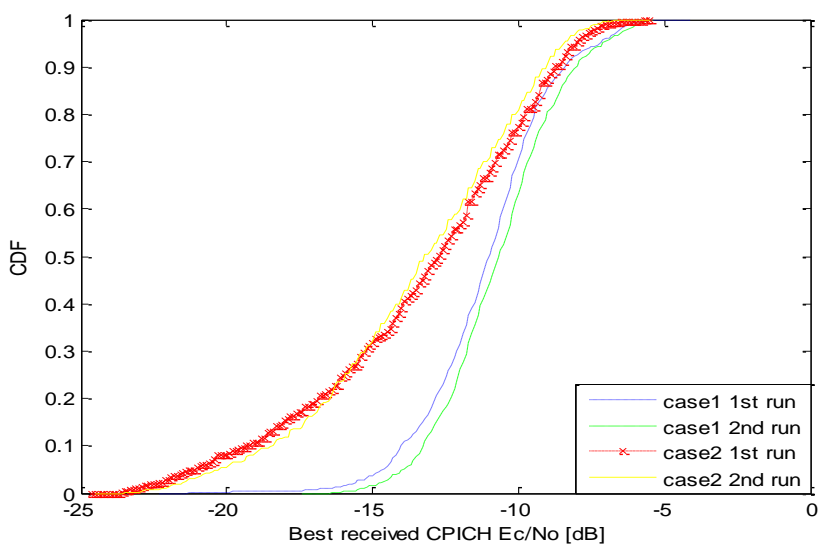

Figure 3. $\mathrm{CDF}$ of Best received $\mathrm{CPICH} \mathrm{Ec} / \mathrm{No}$ in Case 1, and received $\mathrm{CPICH}$ Ec/No from Scr.1 Case 2, respectively

Figure 3 compares the best received $\mathrm{CPICH}$ Ec/No at UE in Case 1 and 2, respectively. Higher $\mathrm{CPICH} \mathrm{Ec} / \mathrm{No}$ values are obtained in Case 1. Considering the CDF value of 0.5, in Case 1 , it corresponds approximately $-11 \mathrm{~dB}$ in $\mathrm{CPICH}$ Ec/No value, which indicates that half of the possibility for $\mathrm{CPICH} \mathrm{Ec} / \mathrm{No}$ values are obtained above $-11 \mathrm{~dB}$; however, in Case 2 , half of the possibility for $\mathrm{CPICH} \mathrm{Ec} / \mathrm{No}$ values are obtained approximately higher than $-14 \mathrm{~dB}$. That is, $3 \mathrm{~dB}$ gain in Case 1 . Furthermore, $5 \%$ of the $\mathrm{CPICH}$ Ec/No values in Case 2 are lower than the performance requirement of $-20 \mathrm{~dB}$ [2].

\section{4) Primary Common Pilot Channel (CPICH) RSCP}

For availability of service for QoS [13], it is essential for UE to stay within the service coverage. If a UE with an in-used VT moved into the "hole"[8] in $3 \mathrm{G}$ service coverage, the UE 
may suffer from e.g. low throughtput, long buffering time, or call drop.

Technically, CPICH RSCP received at UE indicates the coverage probability of WCDMA network. The coverage of a cell in WCDMA network is usually indicated by cell radius, i.e. maximum distance away from the centre of cell where the $\mathrm{BS}$ of that cell is able to provide mobile service to UE. However, in WCDMA, cell coverage is dynamic as balance to its capacity [2]; hence, coverage probability is used. Higher CPICH RSCP measured at UE, higher probability the UE is locating in within network coverage. The relation of $\mathrm{CPICH}$ RSCP value and distance are shown in (2) and (3).

$$
\begin{gathered}
R S C P_{\text {CPICH }}=P_{\text {Tx, CPICH }}-\text { PathLoss }[\mathrm{dBm}] \\
\text { PathLoss }=\frac{A}{r^{-\alpha}}
\end{gathered}
$$

Where, PTx, CPICH is the transmission power as the output of BS antenna; $\mathrm{A}$ is a constant and the value of $\mathrm{A}$ depends on the properties of the BS antenna; $r$ denotes the distance between UE and the BS antenna; $\alpha$ is pathloss exponent, which would be different in value as depending on environment.

In map analysis (Figure 4), the values of on UE along the test path are marked in distinct colors based on the applied color set in Figure 4. High CPICH RSCP values (i.e. >= $60 \mathrm{dBm})$ are obtained more often in the measurements in Case 1 (upper picture in Figure 4) than those in Case 2 (lower picture in Figure 4). Whereas, in Case 2, some best CPICH RSCP value of lower than $-70 \mathrm{dBm}$ have been measured in the UE.

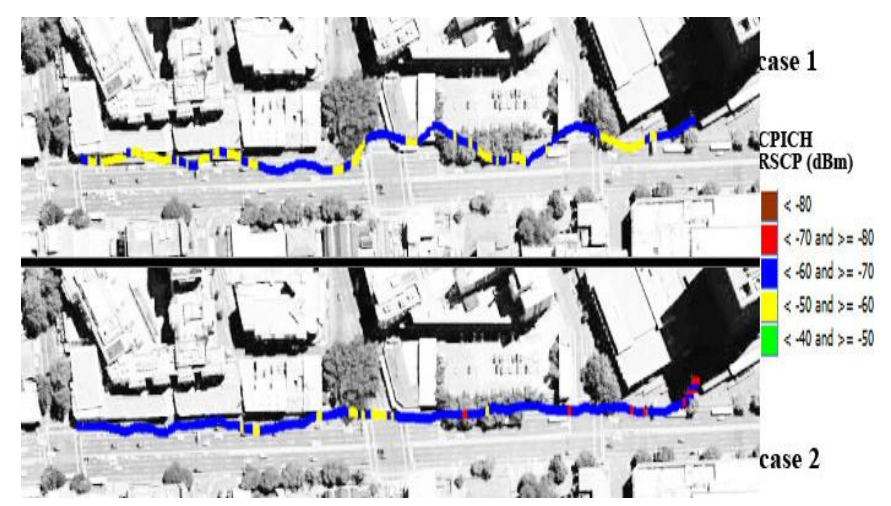

Figure 4. Map analysis of best received CPICH RSCP at UE in Case 1(upper) and Case 2 (lower), respectively. The color set is shown on right-hand side.

Given that the UE in SHO measures CPICH RSCP of multiple BS's, the best received CPICH RSCP is used in data analysis (Figure 5).

According to Figure 5, the CDF curves of measurments in Case 1 shows approximately $4 \mathrm{~dB}$ shift towards higher (left) value of "best received CPICH RSCP" from the CDF curves of Case 2 measurements. That is, an approximately $4 \mathrm{~dB}$ gain has been achieved.

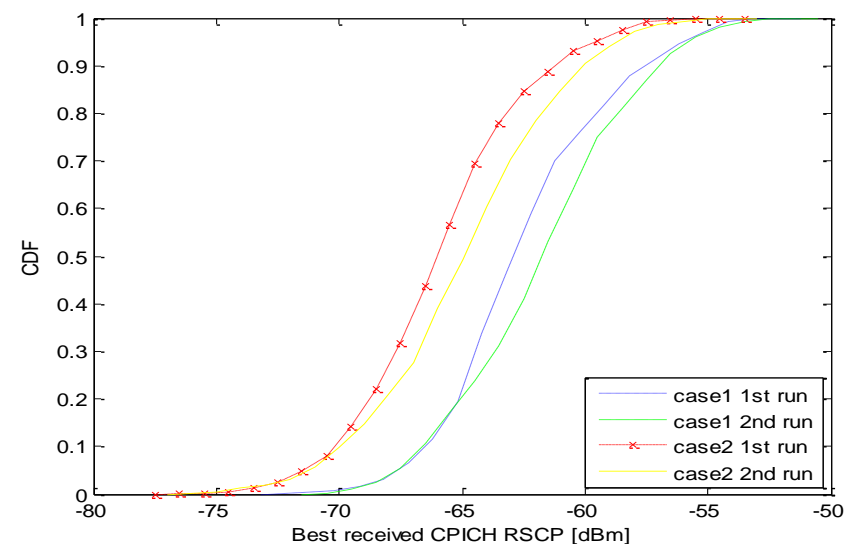

Figure 5. CDF of best received CPICH RSCP on UE from each measurements in Case 1 and Case 2, respectively

5) Downlink "power up" command and. uplink transmission power of $U E$

Another pair of RL quality indicators is from the power control procedures in WCDMA, i.e. the downlink "power up" command from the network and the uplink transmission power of UE. In downlink, the radio network controller (RNC) performs outer loop power control to adjust the received signalto-noise target (SIR target) from the served UE. The adjustment is based on the received signal quality of the UE reported by the serving $\mathrm{BS}(\mathrm{s})$ to RNC. The adjusted SIR target is then sent to the BS(s) from RNC. The SIR target is used as reference for $\mathrm{BS}(\mathrm{s})$ to evaluate the UE should transmit in higher or lower power, and hence sends a "power up" or "power down" command in downlink.

According to the received "power up" or "power down" command, UE adjust its uplink transmission power. In SHO, UE receives power control commands from multiple BS's. In the UE used in this work, the combining algorithm follows any reliable 'power down' command as received [14].

Figure 6 shows the downlink "power up" command received by UE in each measured case, and Figure 7 presents the CDF and PDF analysis of the UE transmission power in each measurements.
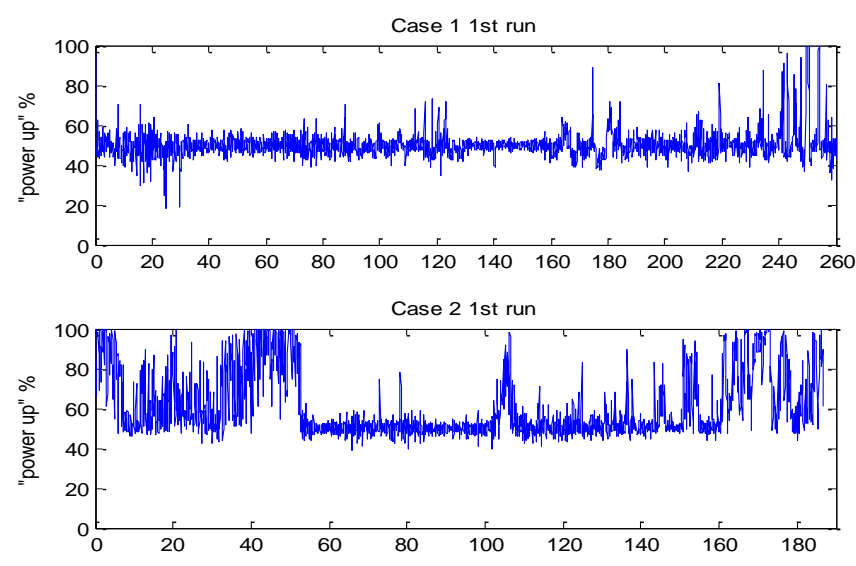

Figure 6. Downlink "Power up" command in Case 1 1st run and Case 2 1st run, respectively. 


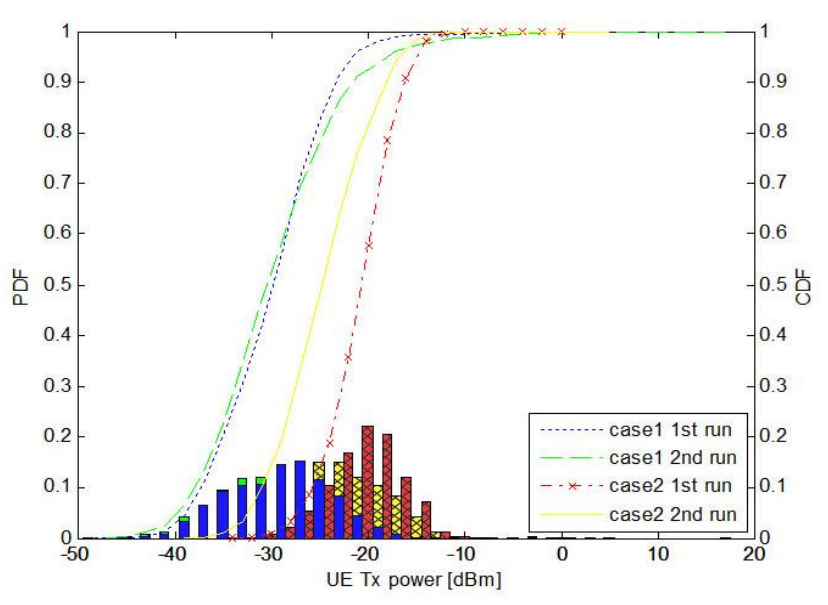

Figure 7. PDF (bars) and CDF (lines) of UE transmission power from each measurements in Case 1 and Case 2, respectively

In Figure 6, the downlink "power up" command in Case 2 (lower figure in Figure 6) occured more frequently than that in Case 1(upper figure in Figure 6). That indicates that the UE is suffering a lot by poor RL condition, seen by the BS. In other words, the transmission of UE has been better received on the network in Case 1.

According to Figure 7, 50\% $(\mathrm{CDF}=0.5)$ of the UE transmission power values are measured lower than approximately $-30 \mathrm{dBm}$ in Case 1 and $-24 \mathrm{dBm}$ in Case 2, based on average of test runs. That is, a reduction of approximately $5.5 \mathrm{~dB}$ in UE transmission power is achieved in SHO scenario. Since the service availability is also limited by the transmission power of UE, the reduction of required transmission power is, in turn, benefit for the UE to be kept service availability from the network when it moving further away from the originally serving BS. Thus, service continuity has been improved.

\section{B. Pilot pollution}

Regarding to Section 1, Pilot pollution is often obtained in cell overlapping areas. However, since the execution of SHO requires cell overlapping (i.e. the area where more than one BS is capable to provide service to UE), pilot pollution is commonly existing in WCDMA networks.

In this work, the criteria of identifying pilot pollution are presented in Table II.

TABLE II. PILOT POLLUTION CRITERIA

\begin{tabular}{|c|c|c|}
\hline Criterions & Symbol & Values \\
\hline $\begin{array}{c}\text { CPICH Ec/No (of other cells) } \\
\text { CPICH Ec/No (of best serving cell) }\end{array}$ & $\zeta_{i, t} *$ & $>=-6 \mathrm{~dB}$ \\
\hline CPICH Ec/No (of best serving cell) & & $<=-8 \mathrm{~dB}$ \\
\hline
\end{tabular}

According to the description of pilot pollution situation, the instantaneous pollution level from each polluting pilot, $\zeta \mathrm{i}, \mathrm{t}$, is defined in this paper as the closeness of other cell $\mathrm{CPICH}$ Ec/No measurements toward the best $\mathrm{CPICH}$ Ec/No in Active Set of the UE (i.e. the best of current serving cells) at time instant $t$. The definition of $\zeta i, t$ is also shown in (5) and (6).

$$
\begin{gathered}
\varsigma_{i, t}=E_{c} / N_{o \text { pollutioni,t }}-E_{c} / N_{\text {obest ASET }, t} \\
\left.\Delta_{i, t}=10^{\varsigma_{i, t} / 10} \quad \text { [linear }\right]
\end{gathered}
$$

Thus, we further define the total level of instantaneous pollution as the sum of effect from all polluting pilots, i.e.

$$
\begin{gathered}
\varsigma_{\text {Total }, t}=\sum_{i=0}^{N_{t}} E_{c} / N_{o_{\text {pollutioni }, t}}-E_{c} / N_{\text {o best ASET }, t} \\
\left.\Delta_{\text {Total }, t}=\prod_{i=0}^{N_{t}}\left(10^{\varsigma_{i, t} / 10}\right) \quad \text { [linear }\right] .
\end{gathered}
$$

By analysing the measurements in this paper, pilot pollution has significant influences on the degradation of RL quality, which are shown in Figure 8 and 9.

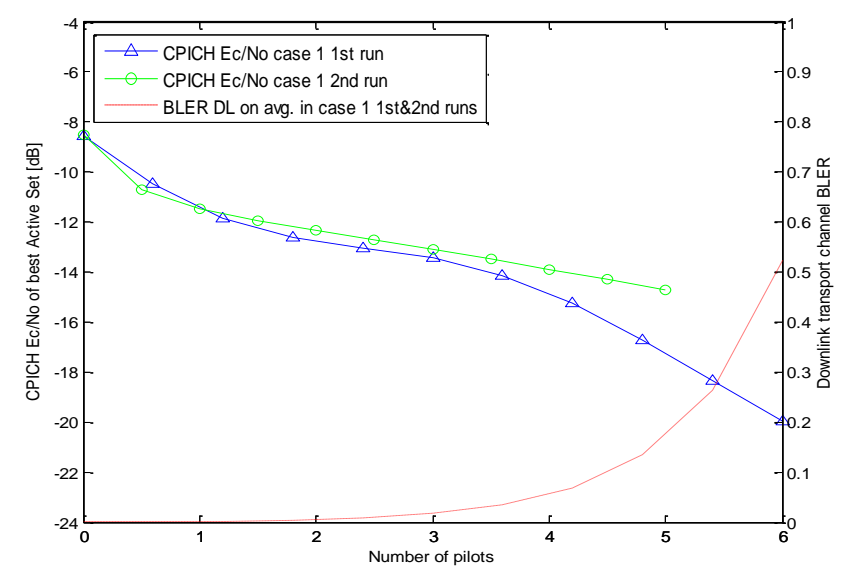

Figure 8. CPICH Ec/No of best received in Active Set decrease when more polluting pilots have been detected; while BLER DL increases only after more than 3 pilots are obtained as polluting pilots

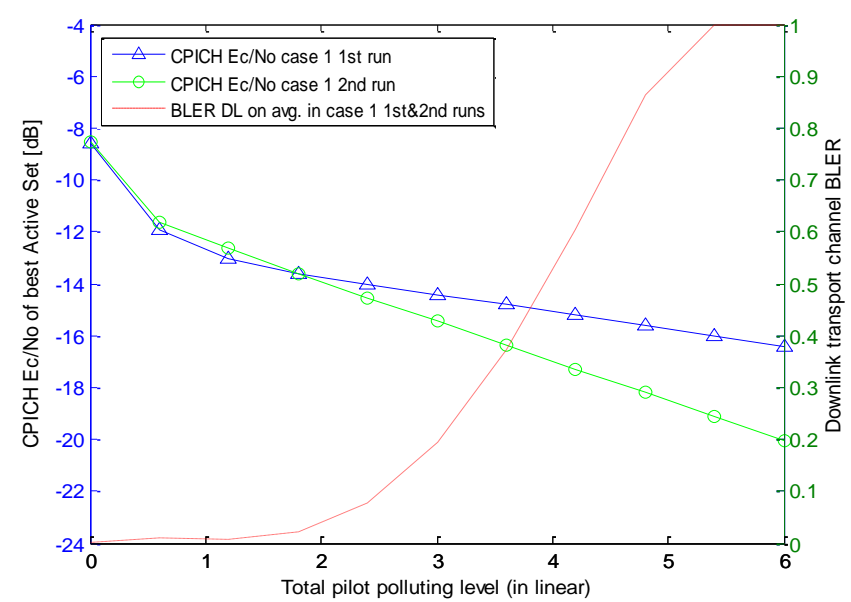

Figure 9. CPICH Ec/No of best received in Active Set decrease when total level of pilot pollution increase. Meanwhile, BLER DL increases significantly along with the rise of total pilot pollution level 
According to Figure 8, the best $\mathrm{CPICH}$ Ec/No in Active Set of the UE decreases significantly when the number of polluting pilots increases. Moreover, note that the Active Set size is set to be maximum 3 cells. It is reasonable to express a particular interest to the point that number of polluting pilots equals to 2 . The CPICH Ec/No has dropped approximately $3.5 \mathrm{~dB}$ when 2 polluting pilots are detected in comparison with the condition that no polluting pilot has been detected.

However, the increase of has become significant only after number of polluting pilots is more than 3 .

In Figure 9, the BLER DL higher sensitivity to the increase of total level of pilot pollution. Yet, the deduction of best $\mathrm{CPICH}$ Ec/No in Active Set is rapid but slightly slower when total level of pilot pollution goes up.

Thus, the negative impact of pilot pollution on RL quality is important. The possible consequences of the impact include: degradation of performance of SHO algorithm [6]; increase of call drop rate; capacity loss [9], etc.

\section{CONCLUSION}

From live measurements, the presence of SHO in WCDMA system brings improvements to RL quality for the UE in using VT, hence improve user mobility and support the network better maintaining QoS.

However, on the other hand, pilot pollution causes the reduction of the RL quality, significantly. Since pilot pollution and SHO usually appear in the same area, i.e. cell coverage overlapping area, it is necessary to mitigate the impact of pilot pollution for getting the full benefit to the overall service quality in WCDMA.

Last but not the least, live measurement provides a practical approach to evaluate performance of commercial network. So it can be compared with theoretical analyses.

\section{REFERENCES}

[1] ETSI, "Universal Mobile Telecommunications System (UMTS); Radio Resource Management strategies," ETSI TR125.922, 2007

[2] H. Holma and A. Toskala, WCDMA for UMTS: HSPA Evolution and LTE, Fourth ed.: John Wiley \& Sons Ltd., 2007

[3] 3GPP, " RRC Protocol Specification," 3GPP TG25.331, 2009.

[4] R. M. Joyce, T. Griparis, L. J. Osborne, B. Graves, and T. M. Lee, "Soft Handover Gain Measurements and Optimisation of A WCDMA Network," 3G Mobile Communication Technologies, 2004. 3G 2004. 5th IEE International Conference, pp. 659- 663, 2004.

[5] T. Isotalo, J. Niemela, J. Borkowski and J.Lempiainen,"Impact of Pilot Pollution on SHO Performance," in Proc. 8th IEEE International Symposium on Wireless Personal Multimedia Communications (WPMC'05), Aalborg, Denmark, Sep. 2005.

[6] M. Malkowski and D. ClaBen, "Performance of Video Telephony Services in UMTS using Live Measurements and Network Emulation," [online] in Wireless Personal Communications:Springer Netherlands, Netherlands: Vol.46, No. 1: 19-32, Jul. 2008.

[7] R. Weber, " Multimedia Performance Assessments in Deployed UMTS Networks," in Proc. 2005 Systems Communications (ICW'5), pp. 166171, Aug. 2005

[8] M. Rahnema, UMTS Network Planning, Optimisation and InterOperation with GSM, Singapore: John Wiley \& Sons (Asia) Pte Ltd., 2008.

[9] 3GPP, " Feasibility study on the mitigation of the effect of the Common Pilot Channel (CPICH) interference at the User Equipment," 3GPP TR25.991, 2002.

[10] R. Kreher, UMTS Performance Measurment.: John Wiley \& Sons Ltd., 2006.

[11] 3GPP, "End-to-end multimedia services performace metrics," 3GPP TR26.944, 2009.

[12] 3GPP, "Physical Layer Measurements (FDD)," 3GPP TS25.215, 2010.

[13] ITU-T, "Definitions of terms related to quality of service," ITU-T E.800, 2008.

[14] 3GPP, "Physical layer procedures (FDD)," 3GPP TS25.214, 2010 